\section{The Theoretical Scenario Experienced in a Real-Life Case of Ulcerative Colitis and Associated Primary Sclerosing Cholangitis: Case 123}

\section{To the Editors,}

We have read the recently published article by Al Draiweesh et al. ${ }^{1}$ studying the safety of combination therapy in patients with inflammatory bowel diseases and primary sclerosing cholangitis (PSC) after liver transplantation with great interest. It is essential to have wide insight regarding biological and antirejection combination therapy. However, there are some small but important details in the publication that need to be clarified by presenting our case.

Theoretically, the typical scenario is ulcerative colitis (UC) and associated PSC leading to liver transplantation. Therefore, current British Society of Gastroenterology guidelines state that colitis should be sought in all patients with PSC using colonoscopy with biopsies. $^{2}$ In our case, liver transplantation was performed on a patient at age 16 after a 6-year history of UC and 2 years of PSC. His colonoscopic pictures consequently revealed endoscopic and histological ulcerative pancolitis with milder activity in the rectum.
According to the current literature, PSC can be accompanied by the rectalsparing type of UC. This coexistence is suggested to result in a lack of response to pharmacotherapies. $^{3} \quad$ Therefore, bowel-selective vedolizumab (VDZ) was started for the patient at age 19; there was no previous biological need during pediatric care. The case series of Al Draiweesh et al. ${ }^{1}$ contains a surprisingly high number of patients with Crohn disease. However, about half of the cases were reclassified after ileal pouch anal anastomosis surgery, probably because of CD-like pouchitis. Coexisting UC and PSC with unusual clinical and endoscopic picture and drug-refractoriness can cause differential diagnostic challenges even for experienced medical centers even after surgery.

Liver transplantation performed before age 18 can be associated with bad drug adherence. Within 6 months after VDZ introduction, the patient required urgent hospitalization because of icterus and threatening rejection because of poor drug intake. Luckily, symptoms alleviated after dose escalation of antirejection therapy and adequate steroid use $(3 \times 1 \mathrm{~g}$ intravenous methylprednisolone). By this time, our patient had already developed recurrent PSC proven by liver biopsy. $\mathrm{He}$ has not experienced serious infections, malignancy, or hepatic failure since then.

Instructively, despite mild clinical activity, continuous endoscopic and histological activity was observed with transfusion-requiring anemia and a subtherapeutic serum VDZ level $(<2 \mu \mathrm{g} /$ $\mathrm{mL}$ ), while the anti-VDZ antibody level also remained low $(<35 \mathrm{ng} / \mathrm{mL})$ after 6 months of therapy.

To summarize, we revealed the importance of studying the current literature in rare cases to ease patient management even by experienced medical centers.

\section{Daniella Pigniczki, MD,* Klaudia Farkas, MD, PhD, * and Tamas Molnar, MD, DSc*}

From the *First Department of Medicine, University of Szeged Faculty of Medicine, Szeged, Hungary

Author contributions: Daniella Pigniczki: data extraction, data analysis, drafting of the manuscript. Klaudia Farkas and Tamas Molnar: supervision of the manuscript.

All authors reviewed and approved the final version of the manuscript.

Conflicts of interest: There is no conflict of interest to be declared.

Address correspondence to: Daniella Pigniczki, MD, First Department of Medicine, University of Szeged Faculty of Medicine, H-6720 Szeged, Korányi fasor 8-10, Hungary (pigniczki.daniella@gmail.com).

\section{REFERENCES}

1. Al Draiweesh S, Ma C, Alkhattabi M, et al. Safety of combination biologic and antirejection therapy post-liver transplantation in patients with inflammatory bowel disease. Inflamm Bowel Dis. 2019. pii: izz244. doi:10.1093/ibd/izz244

2. Chapman MH, Thorburn D, Hirschfield GM, et al. British Society of Gastroenterology and UK-PSC guidelines for the diagnosis and management of primary sclerosing cholangitis. Gut. 2019:68:1356-1378.

3. Horio Y, Uchino M, Bando T, et al. Rectalsparing type of ulcerative colitis predicts lack of response to pharmacotherapies. BMC Surg. 2017;17:59. doi:10.1186/s12893-017-0255-5
C) 2020 Crohn's \& Colitis Foundation. Published by Oxford University Press. All rights reserved. For permissions, please e-mail: journals.permissions@oup.com. doi: 10.1093/ibd/izaa028 Published online 11 February 2020 